\title{
Characteristics of hydrogenated fiber Bragg gratings
}

\author{
C. L. Liou ${ }^{1}$, L. A. Wang ${ }^{1, \star}$, M. C. Shih ${ }^{2, \star \star}$, T.J. Chuang ${ }^{2}$ \\ 1 Institute of Electro-Optical Engineering, National Taiwan University, No. 1, Sec. 4, Roosevelt Rd., Taipei, Taiwan, ROC \\ (Fax: 886-2/365-6327,E-mail: lon@ laser.ee.ntu.edu.tw) \\ 2 Institute of Atomic and Molecular Sciences, Academia Sinica, P.O. Box 23-166, Taipei 10764, Taiwan, ROC
}

Received: 2 September 1996/ Accepted: 8 November 1996

\begin{abstract}
By utilizing both hydrogen loading for photosensitivity enhancement and a phase mask for holographic exposure, we have fabricated fiber Bragg grating with controllable reflectance and bandwidth. The evolution of hydrogen diffusion into a single-mode optical fiber before exposure, and out of the fiber after exposure was characterized, and the results were consistent with the theoretical modeling. The shifting of Bragg wavelength and thermal reliability were found related to the hydrogen behavior inside the fiber. One solution to prevent Bragg wavelength from drifting after the grating was formed and hence to increase the grating's reliability was to anneal it so that the residual hydrogen was forced out of the optical fiber in a short time.
\end{abstract}

PACS: $42.70 ; 42.80$

With the rapid development in telecommunication, optical fibers play an important role in transmission systems as a low-loss and wide-bandwidth medium. However, traditional method of processing the optical signals in such systems have been via interruption of the fiber and subsequent insertion of bulk- or integrated-optic components. For these methods of signal processing, there are several major disadvantages including high device insertion loss, high back reflection, fiber-to-device interfacing problem, and mechanical and thermal stability. As a result, there has been a steady increase in the development of continuous-fiber components capable of performing a variety of essential functions, e.g., wavelength multiplexing and directional switching. The emergence of UVinduced fiber gratings in germanium- doped silica fibers $[1,2]$ has generated significant interest for their numerous applications in optical fiber devices. Light-sensitive fibers offer exciting prospects for the development of such devices as fiber grating assisted semiconductor lasers [3], mode couplers [4], sensors [5], etc. However, it had been a difficult task to fabricate fiber gratings with UV laser sources for their low coherent length until 1993 when Hill et al. [6] proposed a convenient method based on the near-contact exposure through a phase

\footnotetext{
* To whom all correspondence should be addressed

${ }^{\star}$ Current address: Industrial Technology Research Institute, Chutung, Hsinchu, Taiwan, ROC
}

mask. This method has the significant advantage for its much less relaxed requirement on the spatial coherence of exposure light source.

In this paper, we discuss the fabrication of fiber Bragg grating by utilizing hydrogen loading treatment on dispersion shifted fibers and a phase mask for UV light exposure. A diffiusion model is employed to verify the evolution of hydrogen concentration in-diffuse and out-diffuse of an optical fiber. The shifting of Bragg wavelength of a fiber grating which may result in signal instability for practical applications is characterized, and annealing is found effective in preventing such shifting. Finally, the thermal reliability of hydrogenated fiber Bragg gratings is discussed.

\section{Photosensitivity of hydrogenated optical fibers}

\subsection{Hydrogen induced loss in hydrogenated optical fibers}

It is now well known that a germanium-doped silica fiber exhibits excellent photosensitivity in the fabrication of fiber grating. However, the initial fibers used to write fiber grating are restricted to the ones containing high concentration germanium as a dopant. The detection of photosensitivity in germanium-free fibers such as europium-doped [7], cerium-doped [8], phosphorus-doped [9] suggests that the phenomenon does not arise from the presence of a unique chemical dopant. In addition, boron-codoped germanosilicate [10] and tin-codoped germanosilicate optical fibers [11] have also been proved highly successful in producing large index modulation.

In 1993, P. J. Lemaire et al. [12] first described a very effective photo-sensitization method by soaking optical fibers in high-pressure hydrogen at room temperature for a few days [12]. Compared with the non-hydrogen-loaded fibers, the fibers treated in this way exhibit excellent photosensitivity in UV irradiation, typically two orders of magnitude larger [13]. Consequently, this approach is adopted to enhance the photosensitivity of optical fibers prior to UV light exposure in the fabrication of fiber gratings. The fibers we used were of dispersion shifted ones which had quasi-triangular index profiles with core diameters of $5.7 \mu \mathrm{m}$. The maximum measured index difference between core and cladding was about 


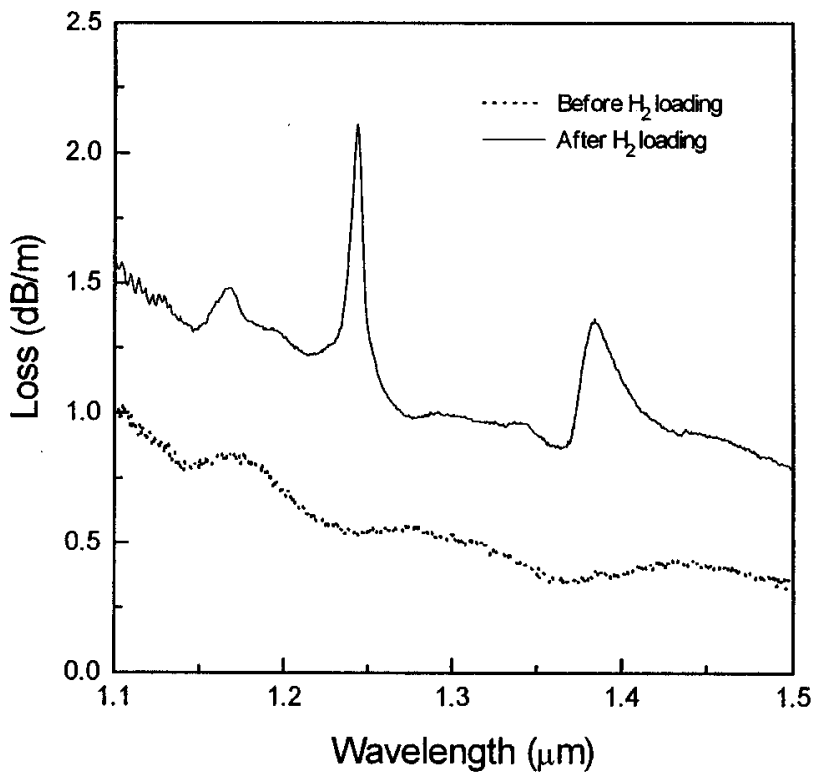

Fig. 1. Loss spectra of an optical fiber before and after $\mathrm{H}_{2}$ loading treatment

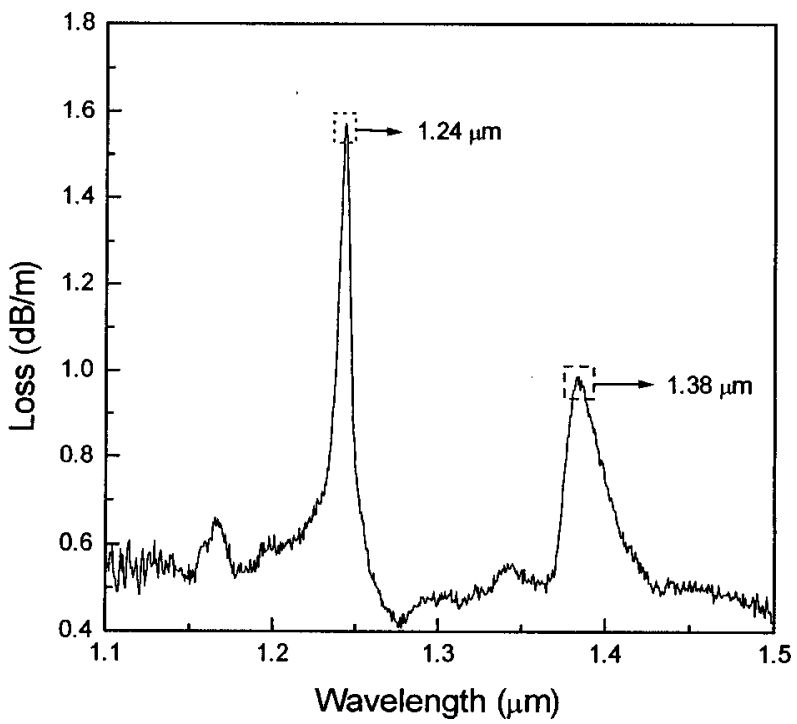

Fig. 2. Difference of the loss spectra in Fig. 1, featuring two main peaks

0.017 in its preform form. Figure 1 shows the loss spectra of an optical fiber before and after hydrogen-loading treatment. Dispersion-shifted fibers which had been loaded for 20 days in the hydrogen chamber at the pressure of $125 \mathrm{~kg} / \mathrm{cm}^{2}$ and temperature of $20^{\circ} \mathrm{C}$ were utilized in the measurement. Subtracting these two curves, it is apparent that there exists two absorption peaks in Fig. 2: one centers at $1.24 \mu \mathrm{m}$ and the other at $1.38 \mu \mathrm{m}$. The loss at $1.38 \mu \mathrm{m}$ is due to the conventional $\mathrm{OH}$ absorption. The one at $1.24 \mu \mathrm{m}$ is however associated with hydrogen [14], and its magnitude is directly proportional to the concentration of hydrogen in the core region of the fiber. Therefore, the magnitude of the absorption peak can be used as an indicator to monitor the concentration of hydrogen in the core. By characterizing the evolution of hydrogen diffusion, the time constant defined in the next section for reaching the saturation level can be obtained. Figure 3 shows the evolution of hydrogen concentration when the optical fibers were

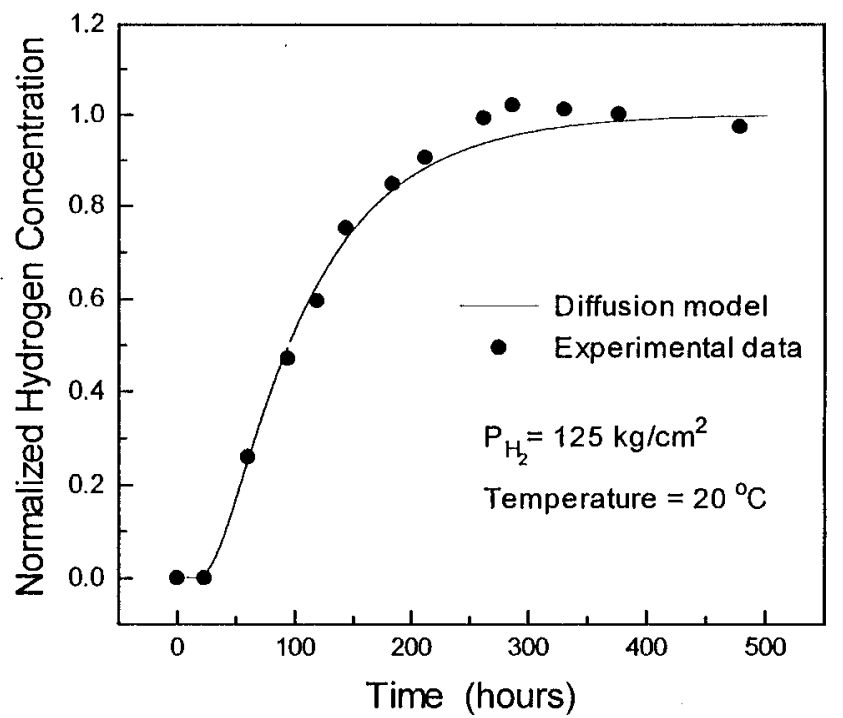

Fig. 3. Evolution of $\mathrm{H}_{2}$ diffusion into optical fibers: the curve is calculated by using the diffusion model and the solid circles are the normalized losses measured at $1.24 \mu \mathrm{m}$

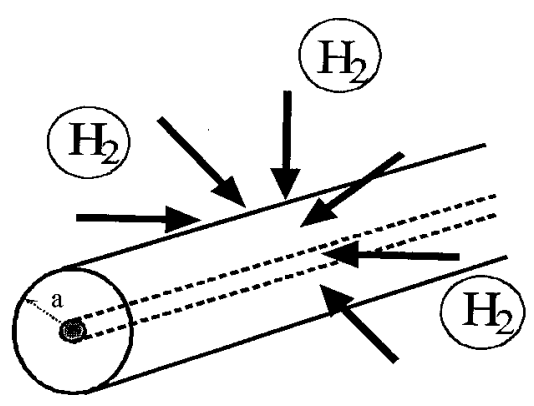

Fig. 4. A schematic diagram of hydrogen diffusion into a cylinder

immersed in hydrogen with the same conditions described previously. The solid circles in the figure represent the measured hydrogen-induced losses at $1.24 \mu \mathrm{m}$ normalized to the saturated value. The line curve is the simulation result obtained by employing the diffusion model, to be described in the following section. The good agreement between the measurement and the simulation clearly suggests that there is a strong correlation between the hydrogen-induced loss and hydrogen concentration.

\subsection{Diffusion Modeling}

Consider a long cylinder in which diffusion is radial everywhere as depicted in Fig. 4. We further assume that core and cladding regions of the cylinder have the same diffusion coefficient for hydrogen owing to the minute difference of the refractive indices. The concentration of hydrogen diffusing into the cylinder is therefore a function of radius, $r$, and time, $t$, only, and can be obtained by solving the diffusion equation [15]

$\frac{\delta C}{\delta t}=\frac{1}{r} \frac{\delta}{\delta r}\left(r D \frac{\delta C}{\delta r}\right)$ 
where $C$ is the hydrogen concentration and $D$ is the diffusion coefficient. Owing to the constant pressure and temperature in the chamber, the hydrogen concentration is therefore a constant value $C_{O}$ outside the surface $(r=a)$. Assume the initial concentration is zero inside the cylinder. The boundary conditions can therefore be described as

$$
\begin{gathered}
C=0 \text { for } 0<r<a, t=0 \\
C=C_{0} \quad \text { for } r=a, \quad t>0
\end{gathered}
$$

With these two boundary conditions, the normalized solution of Eq. (1) can be expressed as

$$
\frac{C(r, t)}{C_{0}}=1-2 \sum_{n=1}^{\infty} \frac{\exp \left(-D \alpha_{n}^{2} t\right) J_{0}\left(r \alpha_{n}\right)}{a \alpha_{n} J_{1}\left(a \alpha_{n}\right)}
$$

where $\alpha_{n}$ 's are the roots of $J_{0}\left(a \alpha_{n}\right)$, and $J_{0}(x)$ and $J_{1}(x)$ are the Bessel function of the first kind of order zero and one, respectively. Since the photosensitivity of a hydrogenated fiber occurs only in its core region, the total amount of hydrogen molecules in the core therefore accounts for the resultant photosensitivity. An integration of Eq. (4) over the core region to obtain the total amount of hydrogen molecules has thus been performed. It is found, however, the difference between the concentration and the total hydrogen amount is negligible for the core radii up to $10 \mu \mathrm{m}$. The hydrogen concentration obtained from Eq. (4) can therefore be used as an indicator of the degree of photosensitivity in a hydrogenated fiber.

According to the above diffusion model, the theoretical curve in Fig. 3 is obtained by employing the diffusion coefficient $D=2.32 \times 10^{-11} \mathrm{~cm}^{2} / \mathrm{s}$ to fit the measured hydrogeninduced losses, and the radius of the cylinder is set to be $62.5 \mu \mathrm{m}$. Thus the evolution of hydrogen diffusion in the glass cylinder can be predicted for a given set of pressure and temperature. The hydrogen concentration along the cylinder axis according to normalized solution equation (4) will reach $95 \%$ of the saturation value in a time given by

$t_{0.95}=\frac{0.6 a^{2}}{D_{H 2}}$

where $a$ is the optical fiber's radius and $D_{H 2}$ is the diffusion coefficient of hydrogen molecular in silica. The time constant $\tau_{H}$ this case may be defined as

$\tau_{H}=\frac{a^{2}}{D_{H 2}}$

The larger the time constant, e.g. obtained by a larger cylinder radius and/or a smaller diffusion coefficient, the more time it takes for a fiber to become saturated. For the results shown in Fig. 3, about 12 days elapsed before $95 \%$ of its saturation value was reached.

On the other hand, equally important is to predict how long the induced photosensitivity can last. This is also related to the hydrogen concentration when a treated fiber is removed from the high-pressure hydrogen chamber. Similarly, with the following boundary conditions

$$
\begin{gathered}
C=C_{0} \text { at } 0<r<a, t=0 \\
C=0 \quad \text { at } r=a, \quad t=0
\end{gathered}
$$

the normalized solution is

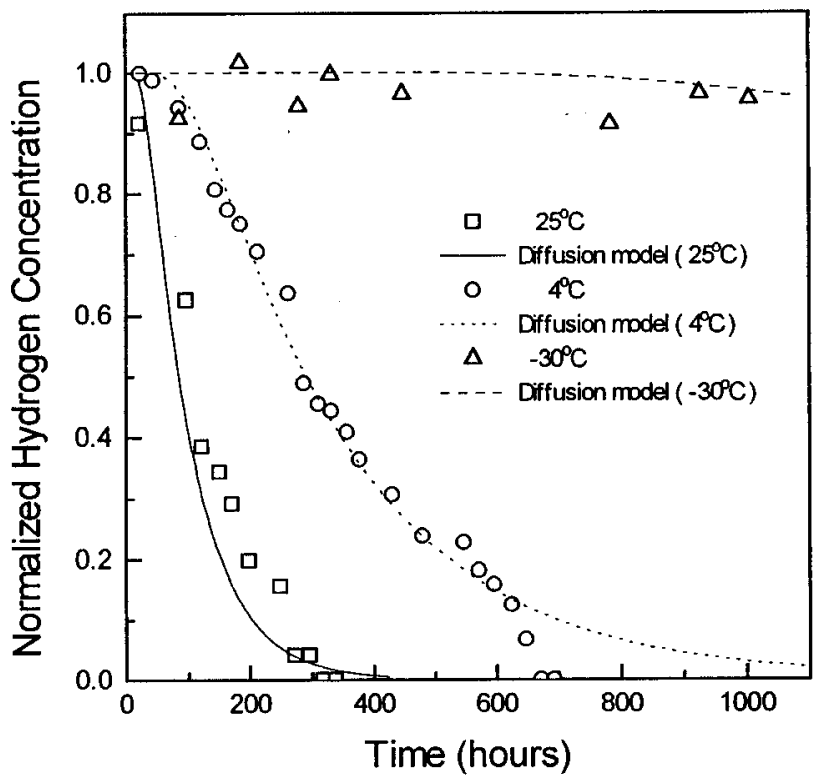

Fig. 5. Evolution of $\mathrm{H}_{2}$ diffusion out of hydrogenated optical fibers at three different temperature $25^{\circ} \mathrm{C}, 4^{\circ} \mathrm{C}$ and $-30^{\circ} \mathrm{C}$. The curves are calculated by using the diffusion model and the symbols are the normalized losses measured at $1.24 \mu \mathrm{m}$

$\frac{C(r, t)}{C_{0}}=2 \sum_{n=1}^{\infty} \frac{\exp \left(-D \alpha_{n}^{2} t\right) J_{0}\left(r \alpha_{n}\right)}{a \alpha_{n} J_{1}\left(a \alpha_{n}\right)}$

The time it takes for the hydrogen concentration outdiffusing to reach $5 \%$ of its final value in the fiber axis is

$t_{0.05}=0.6 \tau$

Figure 5 shows the evolution of hydrogen diffusion out of the fibers that are initially saturated at three different temperatures. The diffusion coefficient $D$ 's used at these temperatures are calculated according to [14]

$D=2.83 \times 10^{-4} \exp \left(\frac{-40.19 \mathrm{~kJ} / \mathrm{mol}}{R T}\right) \mathrm{cm}^{2} / \mathrm{s}$

where $R=8.314 \mathrm{~J} / \mathrm{mol}-\mathrm{K}$ and $T$ is the temperature in Kelvin. The results show that it takes longer time for hydrogen in lower temperature fibers to diffuse out. The photosensitivity of a hydrogenated optical fiber can last for a longer time when kept at a lower temperature, e.g. more than one month at $-30^{\circ} \mathrm{C}$.

\section{Fiber grating fabrication}

The phase mask technique used for writing fiber gratings has the advantages over the traditional holographic method because of its simpler writing setup and more reproducible characteristics. Specifically, the utilization of phase mask relaxes the strict requirement on the coherence of a UV light source. On the other hand, to photoinduce an index change in the fiber core, light absorption must somehow occur. Atkins measured the absorption spectrum in a standard germaniumdoped monomode telecommunication fiber to the wavelength as short as $200 \mathrm{~nm}$ [16]. The results show that a light source in the UV spectral region 228 to $253 \mathrm{~nm}$ is most effective 


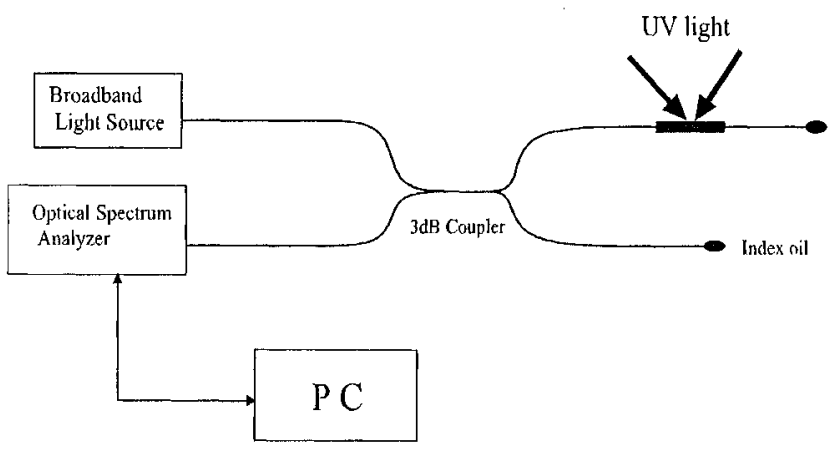

Fig. 6. A schematic diagram of the experimental setup for in situ monitoring fiber Bragg gratings

in photoinducing refractive index changes in such optical fibers. Therefore, we used an excimer laser with wavelength $\sim 248 \mathrm{~nm}$ as light source, and applied the phase mask technique to writing fiber gratings.

\subsection{Experimental setup and measurement results}

A schematic diagram of the experimental setup for in situ monitoring the growth of fiber Bragg grating is shown in Fig. 6. Light from a broadband light source was launched into a fiber which was under UV exposure. A $3 \mathrm{~dB}$ coupler was used to extract the reflected light whose spectrum was in situ monitored. The output ends were dipped with index oil to minimize Fresnel reflection when measuring reflection spectra. The real reflectance of a fiber Bragg grating was recovered by taking the splitting ratio of the coupler in the working spectral region into account. Figure 7 shows the transmission and the reflection spectra of a fiber Bragg grating obtained by irradiating the fiber through a zero-order-nulled phase mask (period = $1078 \mathrm{~nm}$ ) at the fluence of $350 \mathrm{~mJ} / \mathrm{cm}^{2} /$ pulse. A KrF excimer laser $(248 \mathrm{~nm})$ with a repetition rate of $2 \mathrm{~Hz}$ was used to expose an $8 \mathrm{~mm}$-long Bragg grating for a duration of $11 \mathrm{~min}$. The Bragg wavelength of this fiber grating was centered at $1560.2 \mathrm{~nm}$, and its bandwidth measured at full width at half maximum (FWHM) was $0.53 \mathrm{~nm}$. Since the period of the phase mask is known, the effective mode index is estimated to be 1.447 according to the Bragg condition

$\lambda_{B}=2 n_{\operatorname{eff}} \Lambda$

where $\lambda_{B}$ is the Bragg wavelength, $n_{\text {eff }}$ is the effective mode index, and $A$ is the fiber grating period. For a uniform grating, the maximum reflectance can be expressed as [17]

$$
\begin{aligned}
R_{\max } & =\tanh ^{2}(\kappa L) \\
\kappa & =\frac{\pi \Delta n \eta}{\lambda_{B}}
\end{aligned}
$$

where $\kappa$ is the coupling coefficient, $\Delta n$ is the refractive index change, $L$ is the grating length and $\eta$ is the fraction of light propagating in the core. The cut-off wavelength of our fiber was $1199 \mathrm{~nm}$; therefore, the normalized frequency $V$ was equal to 1.848 at $1560 \mathrm{~nm}$. By applying Eqs. (13) and (14) and the calculated $\eta=0.62$ for the dispersion shifted fiber at the normalized frequency, the refractive index change in the optical fiber was estimated to be $2.5 \times 10^{-4}$.

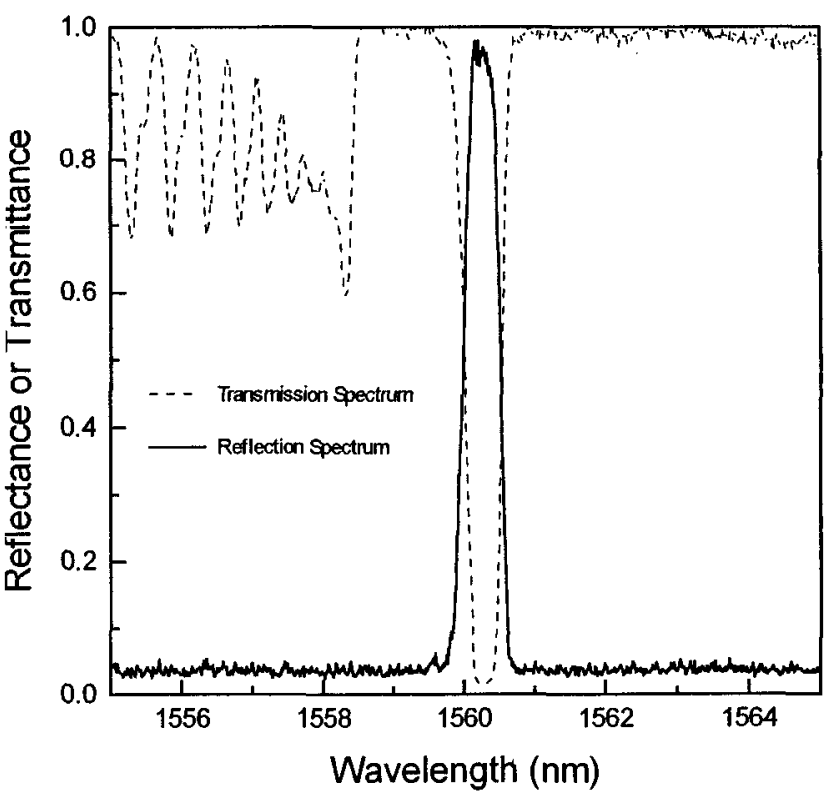

Fig. 7. Transmission and reflection spectra of a typical fiber Bragg grating. The Bragg wavelength $\lambda_{B}$ is centered at $1560.2 \mathrm{~nm}$. For wavelengths less than $\lambda_{B}$, light is coupled to the cladding layer as radiation modes

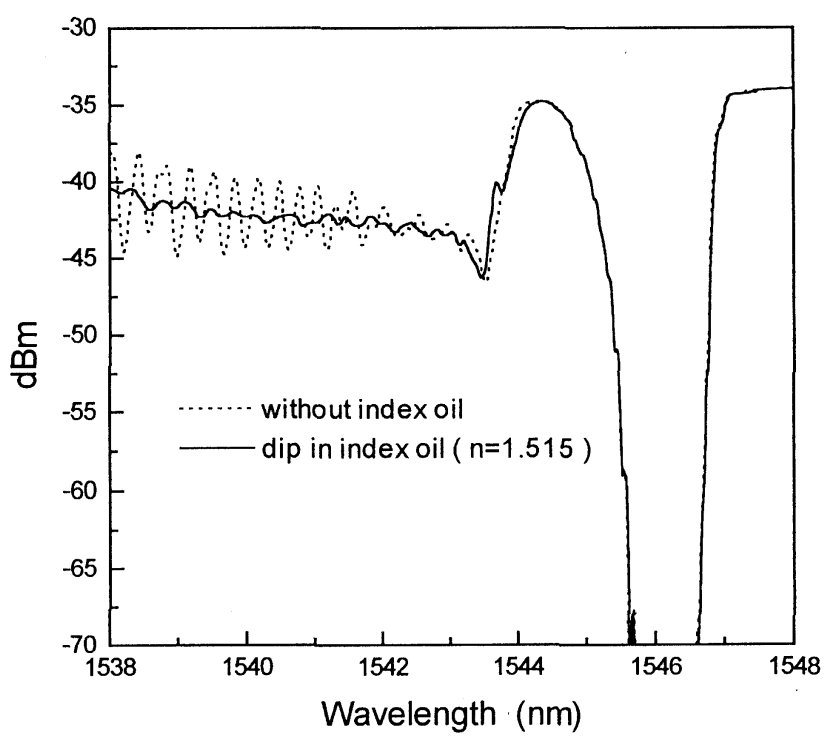

Fig. 8. Transmission spectra of a fiber grating before and after index oil is applied to the fiber grating

In the transmission spectrum of Fig. 7, a pronounced feature appears in the short wavelength side of the Bragg peak. However, as in the reflection spectrum, only the main Bragg peak appears without corresponding features. Since there is no significant variation of UV induced loss in this spectral range, this radiation loss is therefore suggested to be due to light leaving the core edge as a result of the grating inscription. Generally this type of radiation loss has been often observed in the surface relief grating made by physically etching the core of a polished optical fiber [18]. Owing to the absorption of UV light in the core region, in contrast to the transparency of cladding to UV, the refractive index should be changed from the interface between cladding and core, and gradually ex- 
panded in depth when using the side writing technique. Thus, in principle, the structure is similar to the surface relief grating. Theoretically, the light coupling between core and cladding layer on the short wavelength side can be explained by using the model of a cylindrical Fabry-Perot resonance resulting from the light out-coupling into the cladding layer due to the strong grating in the core [19]. The resonant phenomenon as shown in Fig. 8 is more pronounced in a stronger grating, especially serious for the type II grating [20]. After dipping this fiber grating in an index oil ( $n=1.515)$ to eliminate the index mismatch between the cladding and air, the multiple peaks are greatly reduced, leaving only the true radiation mode coupling. It should be noted, however, the wavelength of main Bragg peak is unaffected.

\section{Shifting of Bragg wavelength and thermal reliability}

\subsection{Shifting of Bragg wavelength}

While writing a fiber grating with UV light, it was observed that the Bragg wavelength was shifting toward the longer wavelength side as shown in Fig. 9. Owing to the DC-term of the interference intensity inside an optical fiber, the effective mode index increased with UV exposure. The Bragg wavelength was therefore shifting towards the longer wavelength side. Malo et al. [21] showed that the sign of light-induced refractive index change in a Ge-doped optical fiber was positive measured by using a Mach-Zehnder interferometer when the fiber was irradiated from the side. The result is consistent with ours, i.e. the wavelength also shifts towards the longer wavelength side during exposure. The shifting amount of Bragg wavelength was found linearly increased with time, and the growth of the Bragg reflection became saturated after certain exposure time. In addition, from some limited experiments, it showed that there was a correlation between the rate of Bragg wavelength shifting and the phase mask used for exposure. This suggests that the optical quality of the phase mask might contribute to the controllability of the Bragg wavelength.

After the fiber grating was formed, however, its Bragg wavelength was found gradually shifting in the opposite direction, i.e. toward the short wavelength side. The amount of wavelength shifting depends on several factors such as grating reflectance, hydrogen concentration in the fiber, and exposure dosage of UV irradiation. One of the results is shown in Fig. 10. Also shown is the variation of the absorption peak with time at $1.24 \mu \mathrm{m}$, i.e. an indicator of hydrogen concentration. The trend of the evolution of the hydrogen concentration was apparently related to the Bragg wavelength shifting after the fiber Bragg grating had been formed. Detailed study is currently under investigation. Such shifting will have a serious effect on the devices such as fiber lasers or fiber grating sensors which require stable Bragg wavelength operation. One of the solutions to solve this problem is to anneal the fiber Bragg grating. At higher temperature, hydrogen in a hydrogenated optical fiber will diffuse out at a speed faster than that at room temperature; therefore, the wavelength shifting will be accordingly accelerated to reach a stable state. This technique has been routinely applied in our laboratory to prevent hydrogen induced wavelength drifting.

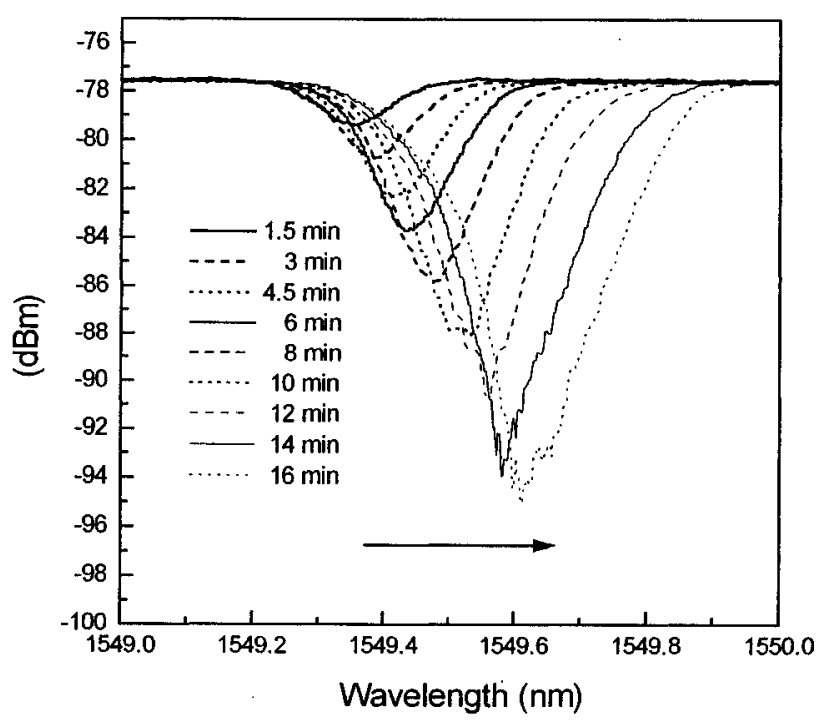

Fig. 9. Transmission spectra of a fiber grating recorded at different exposure time

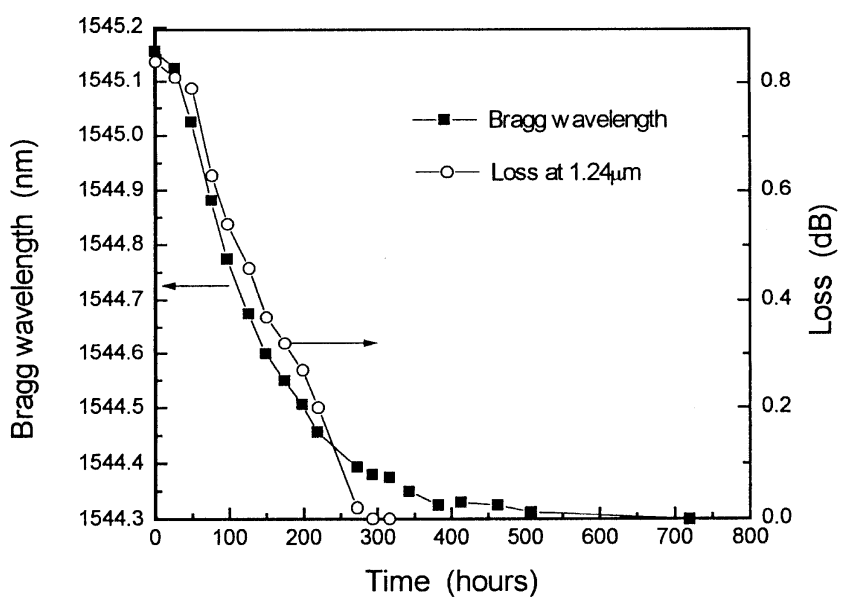

Fig. 10. After the grating is written, its Bragg wavelength is shifted with time as shown in solid squares, and the time varying losses of the fiber are shown in open circles

When the temperature of a fiber Bragg grating changes, its Bragg wavelength shifts because of the thermal expansion effects on refractive index and grating period. However, when we measured a cycle of the evolution of the Bragg wavelength as a function of the grating temperature in the range $25^{\circ} \mathrm{C}$ to $200{ }^{\circ} \mathrm{C}$, a discrepancy of Bragg wavelength was observed in the two curves as shown in Fig. 11a. The measured Bragg wavelength at room temperature after a full cycle was smaller than that before cycling. In addition, the shifting direction of the thermal hysteresis in fiber Bragg grating is consistent with the case of hydrogen out diffusion in optical fibers after UV light exposure as described previously. After annealing this fiber grating at $160^{\circ} \mathrm{C}$ for 3 hours, this thermal hysteresis disappears and no change is detected in the Bragg wavelength after applying again the same temperature rise as shown in Fig. 11b. The fiber gratings can thus have stable optical properties for a long time when used below the annealing temperature. 

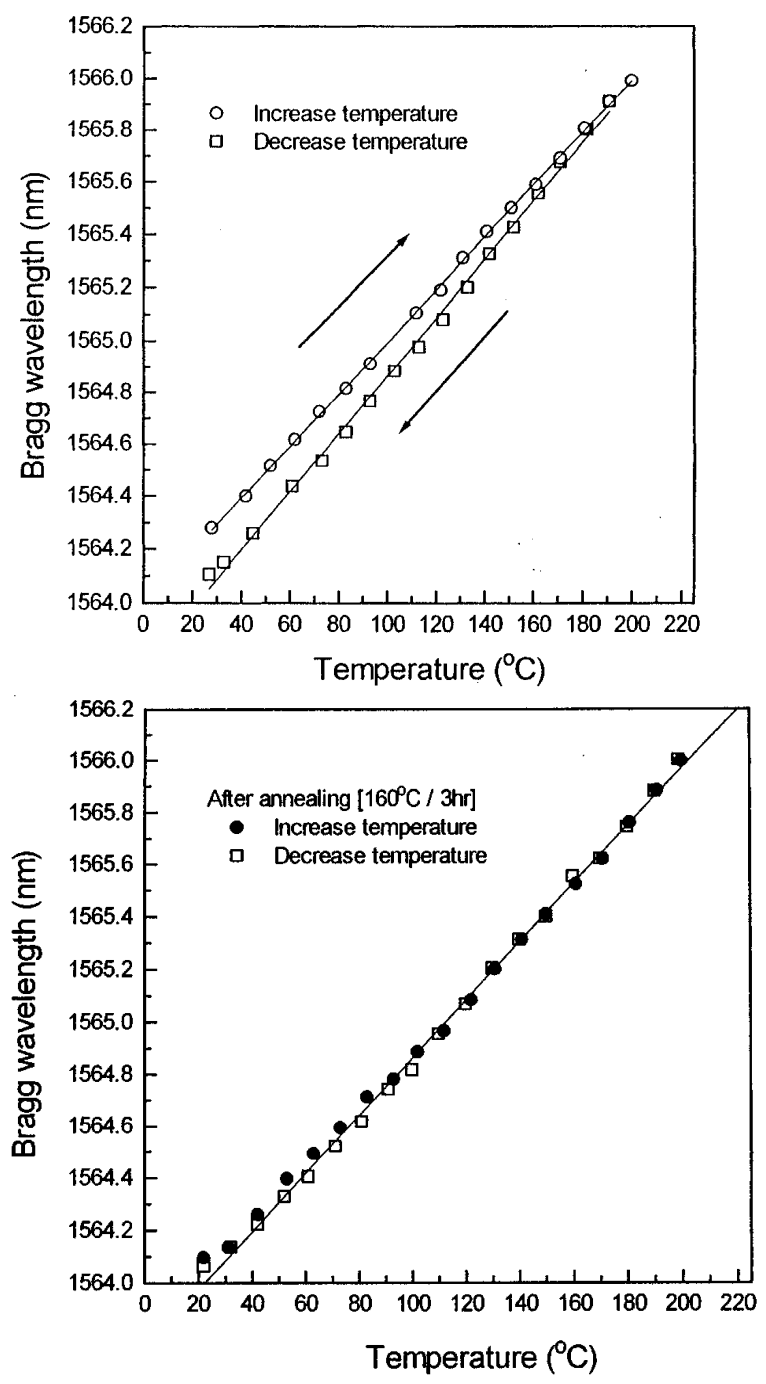

Fig. 11. a Thermal hysteresis of a fiber Bragg grating when temperature cycling is applied; $\mathbf{b}$ After annealing the same fiber Bragg grating, the thermal hysteresis effect is greatly reduced

\subsection{Thermal reliablility}

For practical applications, thermal reliability of a fiber Bragg grating is an important consideration to ensure long-term stability of its performance. We measured the photo-induced index variation with time by annealing fiber gratings in an oven at three different temperatures $100^{\circ} \mathrm{C}, 200^{\circ} \mathrm{C}$, and $300^{\circ} \mathrm{C}$. The gratings written in hydrogenated optical fibers were annealed in 12 days after UV light inscription. We took the reflectance of a fiber grating as $R=1-T$ from the measurement of its transmission spectrum, and then calculated its corresponding coupling coefficient $\kappa$ according to Eq. (13). The time dependence of $\kappa / \kappa_{0}$ at three different temperatures is shown in Fig. 12a where $\kappa_{0}$ is the coupling coefficient measured immediately before annealing. The reflectance of these three fiber gratings was around $90 \%$. The normalized $\kappa / \kappa_{0}$ exhibits the similar trend for all fiber gratings with an initial rapid decay in a short duration followed by a substantially decreasing rate of decay in an extended period, and the decay magnitude increases with the elevated temperature. Additionally, in order to compare the thermal stability for different strength of fiber
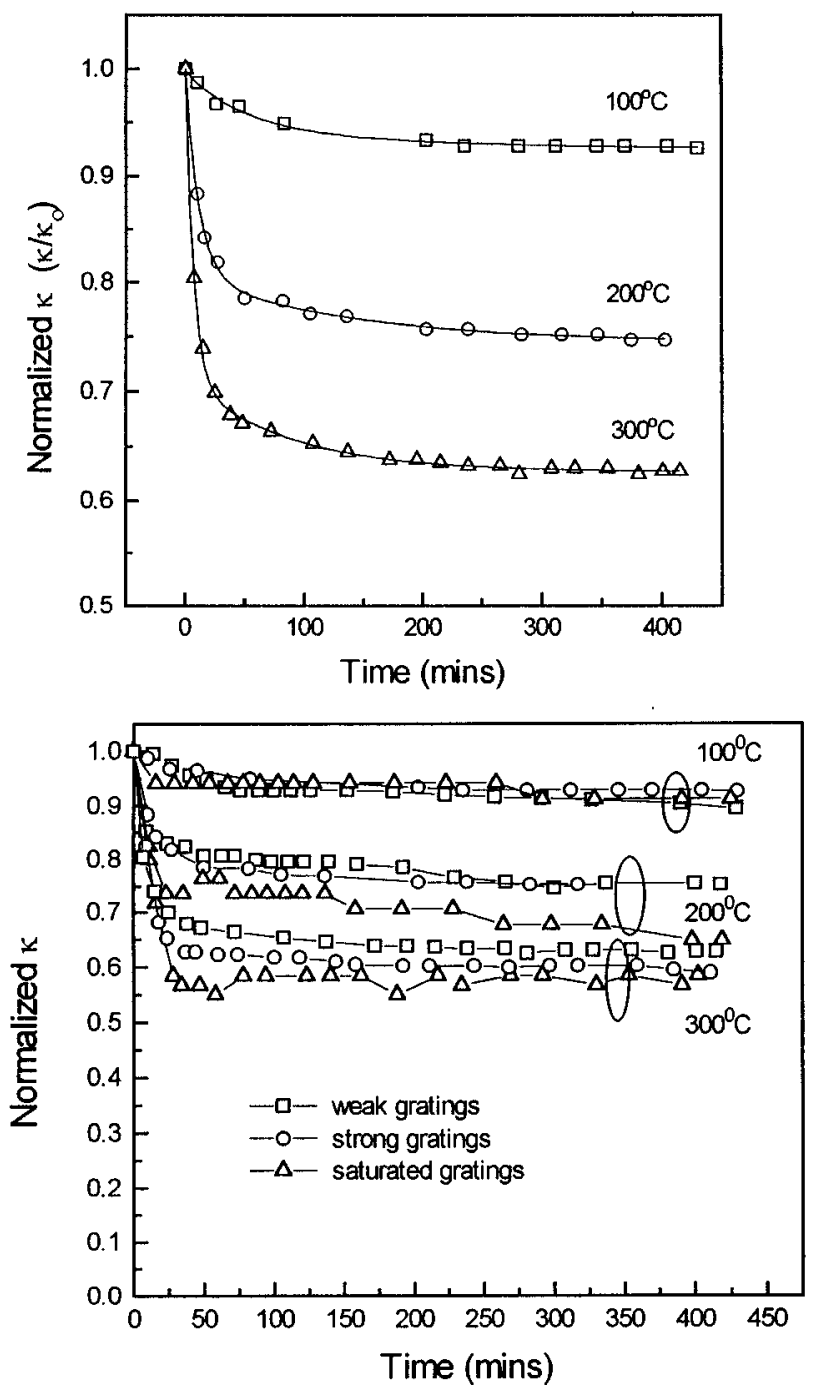

Fig. 12. Thermal reliability at three different temperatures of $100^{\circ} \mathrm{C}, 200^{\circ} \mathrm{C}$ and $300^{\circ} \mathrm{C}$ for top fiber gratings with $R \sim 90 \%$; bottom fiber gratings with three different strengths

gratings, we measured the index variation of another two types of fiber gratings whose reflectance were about $70 \%$ for weak gratings, and nearly $100 \%$ for saturated gratings. The refractive index change for the saturated gratings was alternatively evaluated by taking the bandwidth of $-20 \mathrm{~dB}$ from the horizontal level of the transmission spectrum into the following equation

$\frac{\Delta \lambda_{0}}{\lambda_{0}}=2 \frac{\Delta n}{n_{\mathrm{eff}}}$.

The bandwidth of the saturated gratings according to the definition was about $0.5 \mathrm{~nm}$.

The results incorporating that of the reflectance around $90 \%$ are shown in Fig 12b. The thermal decay curves for the weak and strong gratings are similar, which is consistent with the results reported by R.J. Egan et al. [22]. However, as compared with the weak and strong gratings, the saturated ones had larger decay magnitude. This might be caused by the possible error in the bandwidth measurement due to large magnitude variations of such short bandwidths. It was noted 
that the thermal index variations for fresh fiber gratings measured within 24 hours after the UV light inscription were found no significant difference in comparison with those measured within 2 weeks. From these results, annealing a fiber grating is believed to enhance its performance stability for keeping only the stable portion of the UV-induced refractive index change through the annealing process.

\section{Concluding remarks}

We have fabricated fiber Bragg gratings by utilizing both phase mask technique and hydrogen loading treatment. Hydrogen loading has been proven as an effective method for photosensitivity enhancement. A diffusion model is employed to describe the characteristic of the hydrogen diffusion into and out of optical fibers. In comparison with the experimental data which are obtained by measuring the absorption variations at $1.24 \mu \mathrm{m}$, the simulation results show good agreement with the measurements. The Bragg wavelength of a hydrogenated fiber grating is found shifting during UV light exposure and after grating inscription. A permanent thermal hysteresis is also found in the evolution of Bragg wavelength of a fiber grating as temperature is cycled. The thermal stability for different strength of hydrogenated fiber gratings is characterized. One solution to overcome the problem of Bragg wavelength shifting after writing a fiber grating and hence to increase the grating's reliability is to anneal it so as to force the residual hydrogen out of the optical fiber in a short time.

Acknowledgement. This research was supported in part by the National Science Council, Taiwan, ROC, under the contracts NSC85-2215-E-002-030 and NSC84- 2113-M-001-041.

\section{References}

1. K.O. Hill, Y. Fujii, D.C. Johnson, B.S. Kawasaki: Appl. Phys. Lett. 32, 647 (1978)

2. G. Meltz, W.W. Morey, W.H. Glenn: Opt. Lett. 14, 823 (1989)

3. D.M. Bird, J.R. Armitage, R. Kashyap, R.M.A. Fatah, K.H. Cameron Electron. Lett.27, 1115 (1991)

4. K.O. Hill, B. Malo, K.A. Vineberg, F. Bilodeau, D.C. Jonson, I. Skinner: Electron. Lett. 26, 1270 (1990)

5. L.T. Blair, S.A.Cassidy: Electron. Lett. 28, 1734 (1992)

6. K.O. Hill, B. Malo, F. Bilodeau, D.C. Jonson, J. Albert: Appl. Phys. Lett. 62, 1035 (1993)

7. K.O. Hill, B. Malo, F. Bilodeau, D.C. Jonson, T.F. Morse, A. Kilian, L. Reinhart, O. Kyunghwan: Proc. of Opt. Fiber Comm. PD3-1, 14 (1991)

8. M.M. Broer, R.L. Cone, J.R. Simpson: Opt. Lett. 16, 1391 (1991)

9. T.A. Strasser,A.E. White, M.F. Yan: Proc. of Opt. Fiber Comm. WN2, 159 (1995)

10. D.L. Williams, B.J. Ainslie, J.R. Armitae, R. Kashyap, R.J. Campbell: Electron. Lett. 29, 45 (1993)

11. L. Dong, J.L. Cruz, L. Reekie, M.G. Xu, D.N. Payne: IEEE Photonics Tech. Lett. 7, 1048 (1995)

12. P.J. Lemaire, R.M. Atkins, V. Mizrahi, W.A. Reed: Electron. Lett. 29, 1191 (1993)

13. P.J. Lemaire, A.M. Vengsarkar, W.A. Reed, V. Mizrahi, K.S. Kranz: Proc. of Opt. Fiber Comm. TuL1, 47 (1994)

14. P.J. Lemaire: Opt. Eng. 30, 780 (1991)

15. J. Crank: The mathematics of diffusion, Chap. 5 (Oxford Univ. Press 1975)

16. R.M. Atkin: Opt. Lett. 17, 469 (1992)

17. D.K. Lam, B.K. Garside: Appl. Opt. 20, 440 (1981)

18. C.J. Rowe, I. Bennion, D.C.J. Reid: IEE Proc. 134, 197 (1987)

19. V. Mizrahi,J.E. Sipe: J. Lightwave Technol. 11, 1513 (1993)

20. J.L. Archambault, L. Reekie, P.St.J. Russell: Electron. Lett. 29, 453 (1993)

21. B. Malo, K.A. Vineberg, F. Bilodeau, D.C. Johnson, K.O. Hill: Opt. Lett. 15, 953 (1990)

22. R.J. Egan, H.G. Inglis, P. Hill, P.A. Krug, F. Quellette: Proc. of Opt. Fiber Comm. Tu03, 83 (1996) 